\title{
An unusual case of adult-onset multi-systemic Langerhans cell histiocytosis with perianal and incident thyroid involvement
}

\author{
Ozen Oz Gul', Pinar Sisman', Soner Cander', Erdem Gozden², Meral Kurt³, \\ Ozlem Saraydaroglu4 ${ }^{4}$, Turkay Kirdak ${ }^{5}$, Canan Ersoy ${ }^{1}$ and Erdinc Erturk ${ }^{1}$ \\ 'Department of Endocrinology and Metabolism, Departments of ${ }^{2} \mathrm{Hematalogy}$, ${ }^{3}$ Radiation Oncology, \\ 4Pathology, and 5Surgery, Uludağ University Medical School, Bursa, Turkey
}

\author{
Correspondence \\ should be addressed \\ to $\mathrm{O} \mathrm{Oz} \mathrm{Gul}$ \\ Email \\ drozenoz@gmail.com
}

\begin{abstract}
Summary
Langerhans cell histiocytosis $(\mathrm{LCH})$ is a rare sporadic disease characterized by histiocytic neoplastic infiltration of various organ systems and a wide spectrum of clinical manifestations, ranging from benign and self-limiting to lethal. Herein, we report a rare case of adult-onset multi-systemic LCH in a 36-year-old male patient with an initial perianal presentation and incidental finding of subsequent thyroid gland involvement in the follow-up period. The patient with a history of perianal LCH treated with surgical excision and local radiotherapy was referred to our Endocrinology Department upon detection of hypermetabolic nodular lesions in the left lateral lobe of thyroid gland on positron emission tomographycomputed tomography (PET/CT) scan in the nineth month of follow-up. Current evaluation revealed euthyroid status, a hypoechoic solid lesion of $13 \times 9 \mathrm{~mm}$ in size with irregular borders in the left thyroid lobe on thyroid USG and cytologic assessment of thyroid nodule. The patient was diagnosed with suspected, oncocytic lesion, Hashimoto thyroiditis or LCH. The patient underwent total thyroidectomy and pathological assessment confirmed the diagnosis of Langerhans cell histiocytosis. Assessments in the sixth month of postoperative follow-up revealed euthyroid status with no thyroid tissue remnants or pathological lymph node on thyroid USG. In view of the multifocal lesions indicating multi-system disease, a systemic chemotherapy protocol with combination of prednisone (PRED) and vinblastine (VBL) has been planned by the hematology department.
\end{abstract}

\section{Learning points:}

- Langerhans cell histiocytosis (LCH) shows a wide clinical spectrum and prognosis that ranges from benign and selflimiting single-system disease (with single or multifocal lesions) to a potentially lethal multi-system disease with severe organ dysfunction and death in some cases.

- It has been stated that the diagnosis is often delayed in perianal LCH unless LCH is specifically considered in the etiology, despite the fact that mucosal involvement may precede systemic involvement.

- Our findings support the statement that most of patients with LCH were PET positive at the time of initial diagnosis, while also emphasize the inclusion of this imaging modality as a part of the diagnostic workflow as well as in the setting of treatment response evaluation among adult LCH patients. 


\section{Background}

Langerhans cell histiocytosis (LCH) is a rare sporadic disease that occurs predominantly, but not exclusively, in children (1 per 200000 per year) $(1,2)$ and characterized by a clonal neoplastic proliferation, accumulation and pathological dissemination of histiocytes (Langerhans cell, undifferentiated cell, dendrocyte or macrophage) resulting in the destruction of hard and soft tissues (3).

LCH shows a wide clinical spectrum and prognosis that ranges from benign and self-limiting single-system disease (with single or multifocal lesions) to a potentially lethal multi-system disease with severe organ dysfunction and death in some cases $(2,4)$.

Clinical features of adult-onset LCH are poorly defined owing to its rarity (about 1-2 per million per year), and the treatment remains a challenge as derived to date from pediatric protocols with no specific therapy as well as universally accepted guidelines $(1,2)$.

Involvement of the gastrointestinal tract is very rare in LCH, especially in adults, along with only a few isolated case reports in the literature indicating the involvement of the perianal skin and rectum (5). Likewise, albeit reported to be more common in adults than children, involvement of the thyroid gland in LCH has been considered to be extremely rare, even in the setting of multifocal disease (6).

Herein, we report a rare case of adult-onset multisystemic LCH with an initial perianal presentation and incidental finding of subsequent thyroid gland involvement in the follow-up period.

\section{Case presentation}

A 36-year-old male patient diagnosed with perianal Langerhans cell histiocytosis (LCH) since October 2014 was referred to our Endocrinology department in June 2015 upon detection of hypermetabolic ( $\left.\mathrm{SUV}_{\max }: 7.2\right)$ nodular lesions in the left lateral lobe of thyroid gland on follow-up positron emission tomography-computed tomography (PET/CT) scan performed by the hematology department. The patient had been diagnosed with anal fissure and operated in 2012 during his initial admission to hospital with complaints of rectal pain and constipation. Upon progression of disease with no relief of symptoms and occurrence of rectal wound, the patient had been re-operated in October 2014 and the pathological findings revealed the diagnosis of LCH. In view of continuation of his complaints postoperatively as well as detection of no other focus, he had been administered with local radiotherapy (24Gy 3-dimensional conformal radiotherapy (RT), given as 2 Gy a day, 5 days a week) and has since been followed up by the hematology department.

\section{Investigation}

The patient was referred to our Endocrinology Department in June 2015 with a diagnosis of suspected thyroid malignancy based on systemic PET-CT scan findings. At our department, evaluation of blood biochemistry revealed euthyroid status, while a hypoechoic solid lesion of $13 \times 9 \mathrm{~mm}$ in size with irregular borders was detected in the left thyroid lobe during thyroid USG. Suspected oncocytic lesion, Hashimoto thyroiditis or Langerhans cell histiocytosis was revealed by fine needle aspiration biopsy of the thyroid nodule. In our institution, there is no immunohistochemical study being conducted on cytological samples, so we were not able to determine whether the cytological samples are histiocytic or oncocytic cells. After discussion of the patient at a multidisciplinary team meeting, a total thyroidectomy was performed.

\section{Treatment}

The patient underwent total thyroidectomy and identification of diffuse infiltration of the thyroid tissue by sheets and islands of histiocyte-like cells containing vesicular nuclei with an indented, notched, grooved or 'coffee bean'-shaped appearance, one or two nucleoli, and

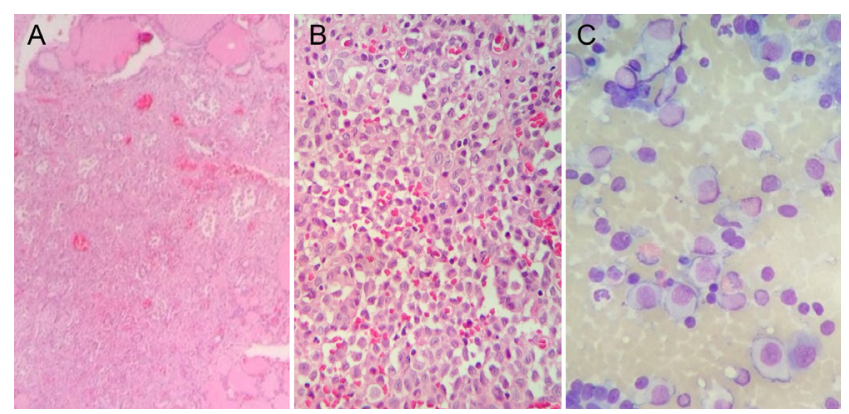

\section{Figure 1}

Langerhans cell histiocytosis diffusely infiltrating the thyroid gland; (A and B) histologic appearance, (C) cytological appearance. (A) Groups of tumor cells located between and separating the thyroid follicles $(H \& E \times 40)$. (B) The tumor cells that are large and contain vesicular nuclei, nuclear fold (groove), pale eosinophilic cytoplasm among eosinophilic leukocytes and erythrocytes (H\&E $\times 400)$. (C) Large cytoplasm and large nuclei of tumor cells between eosinophils and lymphocytes (Giemsa $\times 400$ ). 
abundant lightly eosinophilic to eosinophilic cytoplasm during pathological assessment confirmed the diagnosis of Langerhans cell histiocytosis (Fig. 1). Levothyroxine treatment was initiated upon occurrence of postoperative hypothyroidism with dose adjustments whenever necessary to keep thyroid function in the euthyroid range.

\section{Outcome and follow-up}

Assessments in the 6th month of postoperative follow-up revealed euthyroid status with no thyroid tissue remnants or pathological lymph node on thyroid USG. In view of the multifocal lesions indicating multi-system disease, a systemic chemotherapy protocol with combination of prednisone (PRED) and vinblastine (VBL) has been planned by the hematology department.

\section{Discussion}

Our findings revealed incidental finding of thyroid gland involvement of LCH in an adult patient diagnosed with adult-onset perianal LCH and treated with rectal surgery and local radiotherapy 9 months ago.

Similar to our findings, it has been stated that the diagnosis is often delayed in perianal LCH unless $\mathrm{LCH}$ is specifically considered in the etiology, despite the fact that mucosal involvement may precede systemic involvement (7)

The initial presentation of the disease in our patient included perianal mucosal involvement with no multifocal lesions or involvement of any other organ in extensive workup via systemic PET/CT scan, indicating a single-system LCH.

However, mucocutaneous involvement of adult-onset LCH has been reported to occur more commonly with multi-system disease than with single-organ disease (5), whereas gastrointestinal involvement by LCH among children aged $<2$ years has been considered to be an indicator of widespread multi-systemic disease and poor prognosis in most cases (8).

Low-dose radiation therapy has been considered a treatment choice in multi-system LCH as well as solitary lesions and associated favorable outcome with adequate control of large masses of LCH in the management of localized histiocytosis (1). In adults, systemic chemotherapy has been recommended for those with multifocal single-system or multi-systemic LCH lesions, whereas not for those with localized single-system disease (4).
Indeed, careful observation and local therapy, including curettage or intra-lesional injection of steroids or surgical excision have been recommended for singlesystem LCH to avoid overtreatment as well as shortand long-term complications in patients with low-risk disease (2). Hence, administration of local radiotherapy after surgical excision in our patient with single-system perianal LCH with no evidence on multifocal lesions or involvement of any other organ seems to be consistent with the likelihood of cutaneous involvement of LCH to precede systemic involvement (7).

Given the likelihood of systemic involvement among LCH cases, close follow-up with periodic systemic PET/ CT scans after the initial diagnosis and treatment of perianal LCH in our patient seems notable. PET/CT scan assessment enabled the incidental detection of thyroid gland involvement in our patient only after 9 months of single-system disease and long before the appearance of the clinically overt symptoms such as enlargement of neck mass or a goiter (9). Hence, our findings support the statement that most patients with LCH were PET positive at the time of initial diagnosis, while also emphasize the inclusion of this imaging modality as a part of the diagnostic workflow as well as in the setting of treatment response evaluation among adult LCH patients (10).

Besides given that the course of the disease is unpredictable with self-limiting process in majority of patients along with alternating phase of relapse and remission, our findings emphasize the role of multidisciplinary evaluation in diagnosing and treating adult-onset LCH patients (3).

In systemic reviews of cases with thyroid involvement of LCH, higher frequency of the disease in adults than children, presentation more commonly with diffuse than nodular thyroid enlargement, the presence of euthyroid status rather than subclinical hypothyroidism or hyperthyroidism and lack of thyroidal pain or obstructive symptoms due to a primary LCH goiter in most of cases were reported $(6,11)$. Use of thyroid FNAB was reported to be associated with misdiagnosis of lymphocytic thyroiditis or papillary carcinoma and the misdiagnosis rate has been indicated not to be low $(6,12)$. Hence, a high index of suspicion is needed for accurate diagnosis of LCH in thyroid FNA specimens with the consideration of several other diseases in the differential diagnosis of thyroid cytology specimens such as anaplastic carcinoma, leukemic infiltration of the thyroid, cystic degeneration in goiter, lymphocytic thyroiditis, tuberculosis, non-Hodgkin or Hodgkin lymphoma and chronic granulomatous thyroiditis $(6,12)$. Identification of certain features in 
thyroid FNA specimens such as enlarged and loosely cohesive cells and irregular nuclear contours have been indicated to raise suspicion for LCH involving thyroid (12). Our findings support that, albeit inconclusive, thyroid FNAB is instrumental in initiating diagnostic work up in $\mathrm{LCH}$, while histology remains the most sensitive diagnostic modality (6). Nonetheless, given the likelihood of failure to get ideal biopsy specimens in thyroid nodules for the identification of $\mathrm{LCH}$, it should also be noted that PET/CT is considered a competent diagnostic method for thyroid nodules with inconclusive cytologic results such as LCH involving thyroid (9).

Although optimal treatment of LCH remains controversial due to wide range of clinical spectrum and lack of standard diagnostic and evaluation criteria (3), a combination of prednisone (PRED) and vinblastine (VBL) has been proven to be effective treatment with minimal toxicity and is therefore considered to be the standard initial therapy for all patients in whom systemic therapy is indicated $(1,6)$.

Accordingly, in view of the multi-systemic form of the disease with perianal and thyroid involvement, systemic chemotherapy has been planned in our patient. Notably, thyroid involvement of LCH in adults has been associated with an indolent course with good prognosis after surgical treatment and combination chemotherapy (6).

Incidental detection of thyroid involvement in our patient with history of perianal LCH supports the likelihood of cutaneous involvement to precede multisystemic disease (7) and also the rarity of solitary thyroid gland involvement of $\mathrm{LCH}$, presenting as part of multisystem disease in most cases (6). Indeed, coexistence of LCH and papillary thyroid carcinoma (PTC) was described in past case reports with mutations of the proto-oncogene BRAF almost half of both LCH and PTC patients suggesting an etiologic link between the two diseases $(2,6)$. Association of LCH with chronic lymphocytic thyroiditis or Graves' disease has also been described in the literature (6). Nonetheless, given that direct involvement of the thyroid gland in LCH is extremely rare, even in the setting of multifocal disease (6), our findings indicate a very rare and unique presentation of adult-onset multi-systemic LCH with involvement of perianal mucosa and thyroid but no other systemic foci.

Hence, our findings emphasize the role of prognostic stratification of patients based on the number of involved organs, degree of organ dysfunction and the response to treatment in planning therapies and improving clinical outcomes, increasing survival in multi-systemic disease (2).
In conclusion, we report the clinical experience and management of an adult-onset perianal LCH with an incidental finding of thyroid gland involvement. Our findings underscore the importance of awareness of this rare condition associated with low index of suspicion among practitioners for $\mathrm{LCH}$ as well as the significance of ensuring proper follow-up in adult-onset LCH to rule out systemic disease. Along with implementing a treatment plan matching the prognostic stratification of the patient, multidisciplinary approach and long-term close follow-up with PET/CT scan in the management of adult-onset LCH seem crucial given its rarity and varied presentation.

Declaration of interest

The authors declare that there is no conflict of interest that could be perceived as prejudicing the impartiality of the research reported.

\section{Funding}

This research did not receive any specific grant from any funding agency in the public, commercial or not-for-profit sector.

\section{Patient consent}

Written informed consent has been obtained from the patient for publication of the submitted article.

\section{Author contribution statement}

Primary doctors: Ozen Oz Gul, Pınar Sisman, Soner Cander, Erdem Gozden, Meral Kurt, Ozlem Saraydaroglu, Turkay Kirdak, Canan Ersoy, Erdinc Erturk. Design: Ozen Oz Gul, Pınar Sisman, Soner Cander, Erdem Gozden. Data collection and/or processing: Erdem Gozden, Meral Kurt, Ozlem Saraydaroglu. Literature search: Ozlem Saraydaroglu, Turkay Kirdak, Canan Ersoy, Erdinc Erturk. Writing: Ozen Oz Gul, Pınar Sisman, Soner Cander, Erdinc Erturk.

\section{References}

1 Shevale VV, Ekta K, Snehal T \& Geetanjal M 2014 A rare occurrence of Langerhans cell histiocytosis in an adult. Journal of Oral and Maxillofacial Pathology 18 415-419. (doi:10.4103/0973-029X.151335)

2 Rizzo FM, Cives M, Simone V \& Silvestris F 2014 New insights into the molecular pathogenesis of langerhans cell histiocytosis. Oncologist 19 151-163. (doi:10.1634/theoncologist.2013-0341)

3 Kumar YP, Agrawal J, Mohanlakshmi J \& Kumar PS 2015 Langerhans cell histiocytosis revisited: case report with review. Contemporary Clinical Dentistry 6 432-436. (doi:10.4103/0976-237X.161912)

4 Morimoto A, Shimazaki C, Takahashi S, Yoshikawa K, Nishimura R, Wakita H, Kobayashi Y, Kanegane H, Tojo A, Imamura T, et al. 2013 Therapeutic outcome of multifocal Langerhans cell histiocytosis in adults treated with the Special C regimen formulated by the Japan LCH Study Group. International Journal of Hematology 97 103-108. (doi:10.1007/s12185-012-1245-0)

5 Mittal T, Davis MD \& Lundell RB 2009 Perianal Langerhans cell histiocytosis relieved by surgical excision. British Journal of Dermatology 160 213-215. (doi:10.1111/j.1365-2133.2008.08934.x) 
6 Patten DK, Wani Z \& Tolley N 2012 Solitary langerhans histiocytosis of the thyroid gland: a case report and literature review. Head and Neck Pathology 6 279-289. (doi:10.1007/s12105-011-0321-8)

7 Conias S, Strutton G \& Stephenson G 1998 Adult cutaneous Langerhans cell histiocytosis. Australasian Journal of Dermatology 39 106-108. (doi:10.1111/j.1440-0960.1998.tb01259.x)

8 Lee RG, Braziel RM \& Stenzel P 1990 Gastrointestinal involvement in Langerhans cell histiocytosis (histiocytosis X): diagnosis by rectal biopsy. Modern Pathology 3 154-157. (Review)

9 Cai YF, Wang QX, Ni CJ, Dong SY, Lv L, Li Q, Chen ED \& Zhang XH 2015 A case report: the diagnosis and therapeutic evaluation for a rare disease of langerhans cell histiocytosis involving thyroid. Medicine $\mathbf{9 4}$ e1891. (doi:10.1097/MD.0000000000001891)
10 Derenzini E, Stefoni V, Pellegrini C, Gandolfi L, Broccoli A, Casadei B, Quirini F, Argnani L, Tonialini L \& Zinzani PL 2015 High efficacy of the MACOP-B regimen in the treatment of adult Langerhans cell histiocytosis, a 20 year experience. BMC Cancer 9 879. (doi:10.1186/ s12885-015-1903-8)

11 Attakkil A, Thorawade V, Jagade M, Kar R \& Parelkar K 2015 Isolated langerhans histiocytosis in thyroid: thyroidectomy or chemotherapy? Journal of Clinical and Diagnostic Research 9 XD01-XD03. (doi:10.7860/JCDR/2015/12313)

12 Pusztaszeri MP, Sauder KJ, Cibas ES \& Faquin WC 2013 Fine-needle aspiration of primary Langerhans cell histiocytosis of the thyroid gland, a potential mimic of papillary thyroid carcinoma. Acta Cytologica 57 406-412. (doi:10.1159/000348801)

Received in final form 13 October 2016

Accepted 12 January 2017 